\title{
Alte Muster durchbrechen
}

\section{Julia Rippstein}

Redaktorin Schweizerische Ärztezeitung

\author{
Die Schweiz ist im Hinblick auf die interprofessionelle Ausbildung der Gesund- \\ heitsberufe im Rückstand. In den letzten Jahren wurden jedoch verschiedene Pro- \\ jekte ins Leben gerufen - und dies mit Erfolg. Eins davon ist die Zürcher inter- \\ professionelle klinische Ausbildungsstation. Die Schweizerische Ärztezeitung hat \\ eine Gruppe von Studierenden einen Tag lang begleitet.
}

10.30 Uhr: Im Büro der Klinik für Traumatologie des Universitätsspitals Zürich (USZ) herrscht eine geschäftige Atmosphäre. Es wird Papierkram erledigt, Patientendossiers werden auf den neusten Stand gebracht. Eine junge Frau betritt den Raum und fragt ihre Kolleginnen und Kollegen höflich, aber bestimmt: «Darf ich euch kurz stören? Ich habe eine Frage zu diesem Patienten: Hat er bereits mit der Handrehabilitation begonnen?» Sofort wird von den Bildschirmen aufgeblickt, und die Antwort kommt wie aus der Pistole geschossen: «Ja, er hat bereits mit der Therapie begonnen.» Eine auf den ersten Blick banale Szene. Und doch spielt sie sich nicht in einem gewöhnlichen Büro ab:

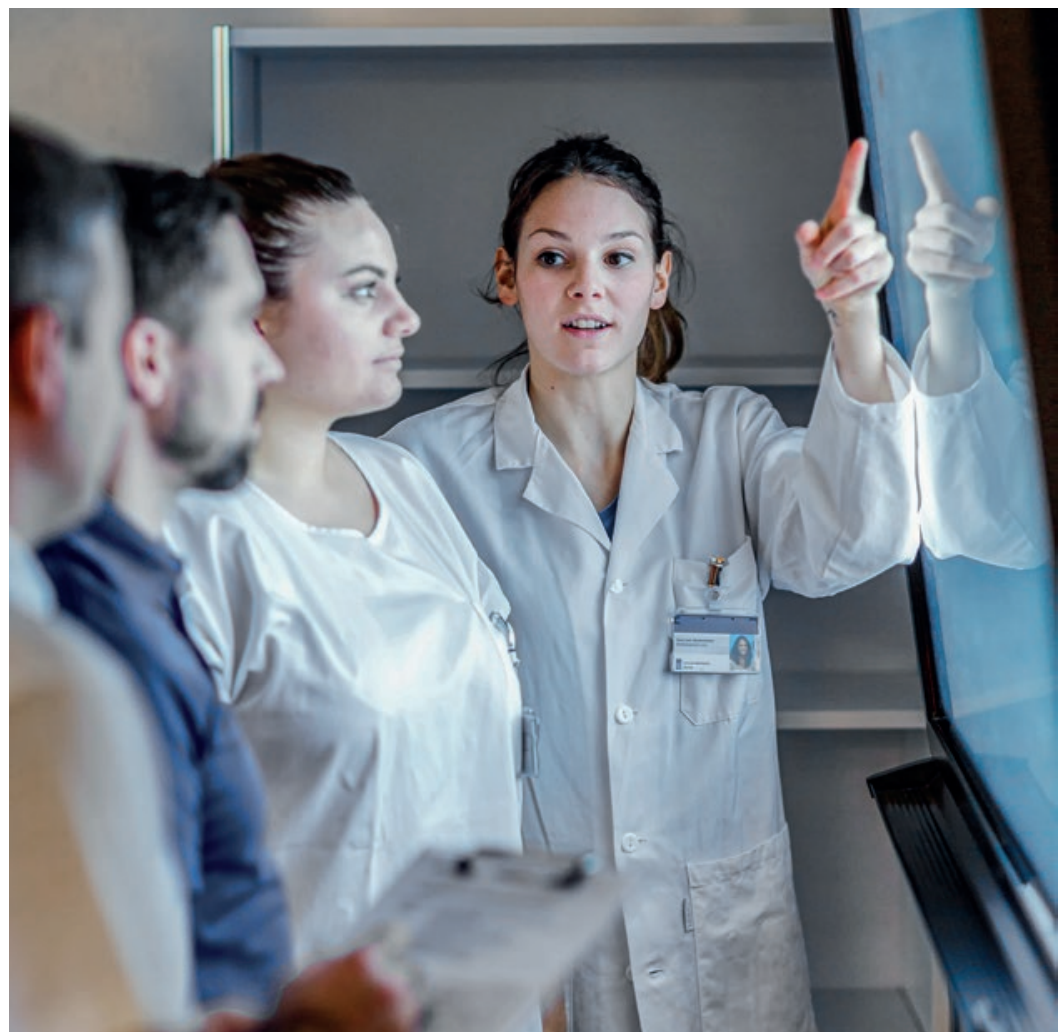

Ausbildungsprojekte wie ZIPAS ermutigen die Studierenden, so viel wie möglich miteinander zu kommunizieren (Foto 2019 aufgenommen: (c) USZ/Nicolas Zonvi).
Hier treffen sich Studierende der Medizin, Pflege, Physiotherapie und Ernährungsberatung, die unter der Aufsicht von «Facilitators» während mehrerer Wochen selbständig Patientinnen und Patienten betreuen und dabei lernen, interprofessionell zu arbeiten. Dieses Konzept hat einen Namen: die Zürcher interprofessionelle klinische Ausbildungsstation (ZIPAS). Das im Herbst 2019 gestartete Projekt basiert auf dem seit fast dreissig Jahren bewährten schwedischen Modell und richtet sich an Studierende aus den Gesundheitsbereichen, die sich in der Endphase ihrer Ausbildung befinden. Das Ziel: Barrieren zwischen den verschiedenen Gesundheitsberufen abbauen, die jeweiligen Aufgaben kennen- und verstehen lernen und vor allem einüben, wie man zusammenarbeitet und wie diese Zusammenarbeit verbessert werden kann. Kurzum: die Interprofessionalität im Alltag der Gesundheitsberufe verankern - und zwar bereits in der Ausbildung.

\section{Büro teilen: immer noch ein Tabu}

Auf die Frage der jungen Frau, die kurz vor dem Abschluss zur Pflegefachfrau steht, folgt ein spontanes «Update» über die drei Patienten, die von den Studierenden betreut werden. "Wer betreut welche Person? Wer braucht Hilfe? Wo gibt es Probleme?», fragt Christian, der ebenfalls angehender Pflegefachmann ist sowie Koordinator des Tages. Die Atmosphäre ist entspannt und herzlich, jeder hört aufmerksam zu und steuert sein Wissen bei. «Dieser Patient wird zunächst nicht operiert, aber wir müssen seinen Fall neu beurteilen», sagt Tobias, ein Medizinstudent.

Dass sich Ärzteschaft und Pflegende ein Büro teilen und sehr offen und konstant miteinander reden, ist in der Realität noch tabu, betont Andrea Bärlocher, Bildungsverantwortliche ZIPAS. Es wäre zwar sehr einfach umzusetzen, wie sie findet, erfordere aber einen Kulturwandel. Dieser Wandel wird von den ZIPAS-Studierenden getragen, für die Interprofessionalität eine 
Selbstverständlichkeit ist. «Der interprofessionelle Aspekt war mir schon immer bewusst, aber seit ich auf der Ausbildungsstation bin, achte ich stärker auf die Aufgaben der anderen Berufsgruppen. Wir unterstützen uns gegenseitig und lernen viel voneinander", sagt Tobias. Für Frank Schäfer, Oberarzt in der Klinik für Traumatologie und Facilitator, kommt die Veränderung dank Projekten wie ZIPAS: «So werden alte Muster durchbrochen.»

Eine Physiotherapie-Studentin tritt ins Büro und fragt schüchtern: «Kann der Patient mit dem Gips eine Last tragen?» Ein Medizinstudent antwortet: «Nein, zunächst keine Belastung, aber ich denke, er kann die
Gelenke bewegen.» Die junge Physiotherapeutin runzelt die Stirn: «Denkst du es oder weisst du es?» Als der angehende Arzt seine Ungenauigkeit bemerkt, korrigiert er sich: "Ich weiss es, wir haben es bei der Operation gesehen.» Ausbildungsprojekte wie ZIPAS ermutigen die Studierenden, so viel und so klar wie möglich miteinander zu kommunizieren. "Oftmals verhindert der Alltagsstress einen offenen Dialog, so dass heikle Themen verdrängt werden und die Zusammenarbeit im Team leidet», sagt Andrea Bärlocher. Das wirkt sich auf die Patientenversorgung aus: 70\% aller medizinischen Fehler sind auf mangelnde Kommunikation und unzureichende Zusammenarbeit zurückzuführen [1].

\section{Fünf Fragen an PD Dr. med. Bastian Grande, Medical Director des Simulationszentrums am USZ}

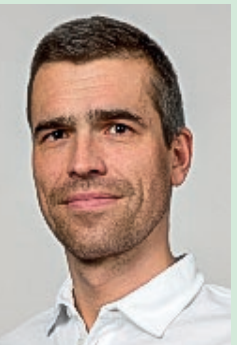

Das Simulationszentrum, das Sie leiten, bietet Trainings speziell für interprofessionelle Teams an. Was sind die Vorteile solcher Trainings?

Wir konnten nachweisen, dass die Leistung eines Teams steigt, wenn es interprofessionelles Arbeiten übt. Die Teammitglieder fühlen sich dadurch sicherer, weil sie wissen, was die anderen tun, und keine Angst haben, untereinander auch manchmal heikle Themen anzusprechen. Dadurch können Hierarchien abgebaut, kann gewaltlos kommuniziert und können Vorurteile gegenüber anderen Berufen überwunden werden. Es schafft Verständnis dafür, dass alle, mit denen man zusammenarbeitet, kompetent sind und sich bestmöglich um das Wohl der Patientin oder des Patienten kümmern wollen.

\section{Warum ist es so wichtig zu wissen, was andere tun? Besteht nicht die Gefahr von Schnittstellen?}

Wenn jeder eingeübt hat, auch die Aufgaben der anderen zu machen, dann weiss jeder, was zu welchem Zeitpunkt zu tun ist. Alle können jederzeit einspringen, wenn jemand Hilfe braucht. Es gibt viele Überschneidungen zwischen den Gesundheitsberufen, so z.B. in der Anästhesie. In diesem Bereich führt das Pflegepersonal ärztliche Tätigkeiten durch. Wichtig ist, zu kommunizieren und die Rollen zu klären, sprich wer macht was. In der Physiotherapie kann die Ärztin oder der Arzt auch Patientinnen und Patienten mobilisieren, muss dies aber klar mit der Physiotherapeutin bzw. dem Physiotherapeuten absprechen: Wie weit greift wer ein, wann ist eine physiotherapeutische Behandlung sinnvoll? In der Interprofessionalität gibt es kein Regelbuch, jede Situation ist anders und muss zwischen den beteiligten Personen ausgehandelt werden.

Es handelt sich um Trainings. Kann das Gelernte im stressigen Alltag wirklich umgesetzt werden?
Ein wichtiger Punkt ist der Transfer des Wissens in die Praxis. Dies geschieht durch Gespräche und Briefings mit denjenigen, die noch nicht über dieses Wissen verfügen. Das Argument «Wir haben keine Zeit» ist eigentlich unberechtigt: Es hat sich gezeigt, dass das gemeinsame Nachdenken über die Zusammenarbeit im Team schneller und effizienter geht als ein Debriefing über eine Patientin oder einen Patienten. Es werden auch heiklere Themen angesprochen. Jeder geht mit einem klareren Kopf und manchmal auch mit einem leichteren Herzen nach Hause.

\section{Was ist mit älteren Kolleginnen und Kollegen, die nicht in} Interprofessionalität geschult worden sind? Machen sie mit? Ich habe die Erfahrung gemacht, dass Kolleginnen und Kollegen unter den starren Strukturen gelitten hatten und froh waren $\mathrm{zu}$ sehen, dass die Zusammenarbeit zwischen den Berufsgruppen auch anders funktionieren kann. Andere sagen: "Ja, das ist schön und gut, aber man muss auch zeigen können, dass es etwas bringt.» Es handelt sich schlicht um einen Kulturwandel, der stattfinden muss. Ich selbst hatte während meiner Ausbildung nie einen Kurs zu diesen Themen. Es wird noch einige Zeit dauern, bis die Interprofessionalität für alle Fachpersonen der Gesundheitsberufe eine Selbstverständlichkeit sein wird.

\section{Im Vergleich zu Ländern wie Schweden und Kanada hinkt die Schweiz in Bezug auf die interprofessionelle Ausbildung hinterher. Woran liegt das?}

Meiner Meinung nach fehlt der politische Wille dazu und damit auch die Strukturen, die solche Ausbildungen fördern. Die FMH könnte hier eine wichtige Rolle spielen. Zudem gibt es keine Fachgesellschaft, die sich speziell dem interprofessionellen Training widmet. Die Fachgesellschaften arbeiten unabhängig voneinander. Ein frappantes Beispiel: Die Broschüren, die nach Kongressen verteilt werden, sind in zwei Teile gegliedert, einen für die Pflege und einen für die Ärzteschaft. Das ist quasi der Gipfel, wenn man bedenkt, dass wir täglich zusammenarbeiten. 


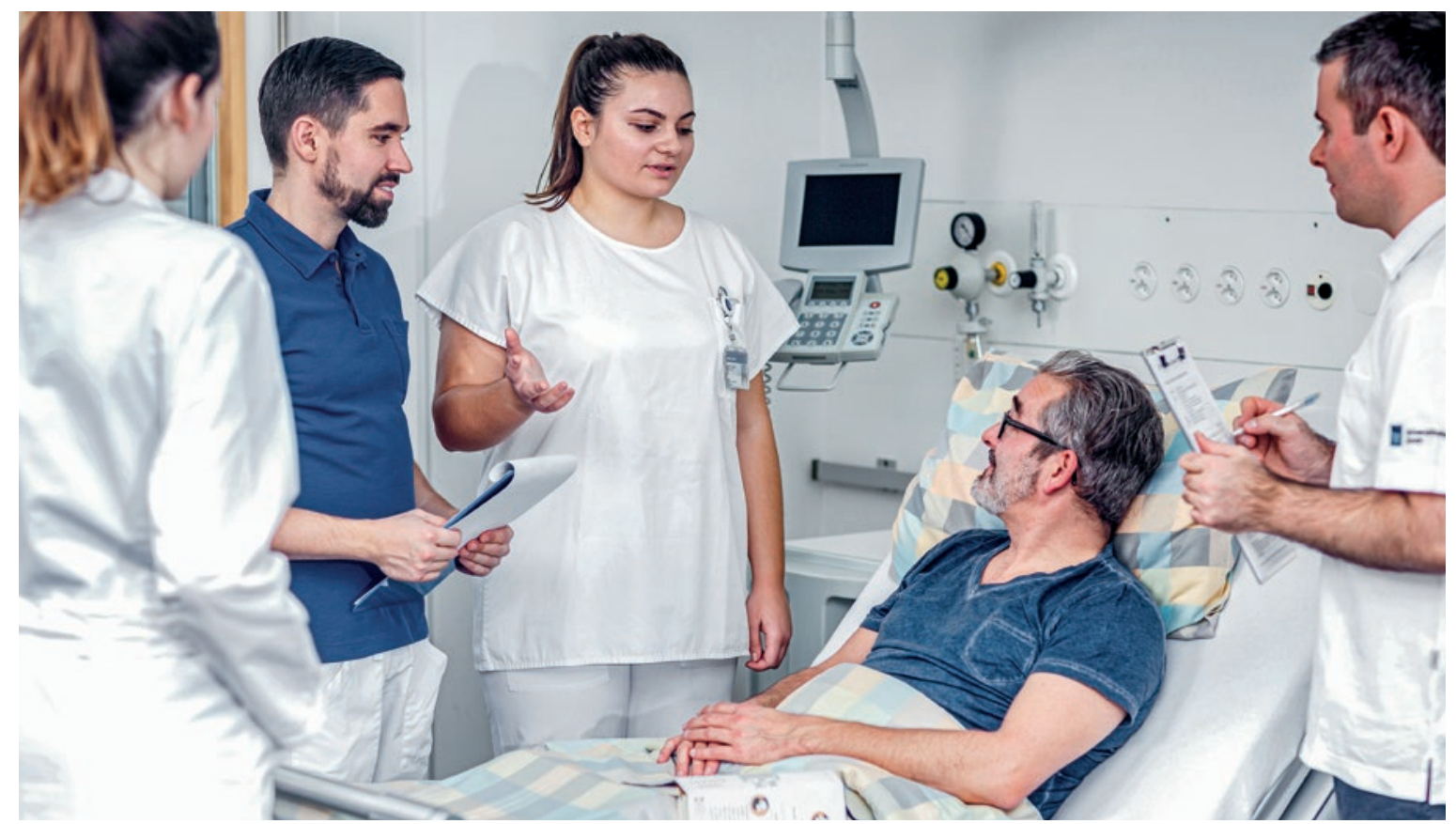

Auf der ZIPAS betreuen Gesundheitsstudierende unter der Aufsicht von «Facilitators» selbständig Patientinnen und Patienten und lernen dabei, interprofessionell zu arbeiten (Foto 2019 aufgenommen: (c) USZ/Nicolas Zonvi).

\section{«Fühlt sie sich schlecht integriert?»}

Als die angehende Physiotherapeutin gegangen ist, schauen sich die anderen Studierenden an: «Sie hat das Gefühl, dass sie uns stört, aber wieso? Es stimmt schon, dass wir uns unter Pflegenden und Ärzten stark austauschen und weniger mit Physiotherapeuten und Ernährungsberatern, die nur sporadisch bei uns sind und sich dadurch vielleicht nicht gut integriert fühlen. Vielleicht ist das ein Punkt, der bei der Reflexion angesprochen werden sollte?» Alle nicken. Die «Reflexion» ist ein fester Lernbestandteil bei ZIPAS und findet täglich statt: Es geht darum, sich über die gelebte interprofessionelle Zusammenarbeit Gedanken zu machen, zu sehen, wann sie gut oder weniger gut funktioniert hat, solche Situationen zu analysieren, zu verstehen, warum jemand so oder so reagiert hat, und die Kommunikation und die Teamarbeit zu verbessern.

Doch vorher machen die Studierenden die Visite bei den drei Patienten, die sie derzeit betreuen. Bevor die Gruppe ins Zimmer geht, trifft sie sich, begleitet vom Facilitator, mit der Assistenzärztin auf dem Flur für das Briefing. Der Fall eines jungen ausländischen Patien-

\section{Schwerpunktserie Interprofessionalität}

Die interprofessionelle Zusammenarbeit von Fachpersonen aus verschiedenen Gesundheitsberufen gilt als wichtiges Mittel, um den Herausforderungen im Gesundheitswesen zu begegnen. Aber wie weit ist die Schweiz in diesem Bereich tatsächlich? Welche Hürden und welche Chancen gibt es? In unserer Schwerpunktserie betrachten wir das Thema aus unterschiedlichen Perspektiven. ten, der kaum Deutsch spricht, beschäftigt das Team. «Wir haben ihm mehrmals erklärt, was er hat und warum er im Spital bleiben muss. Gestern war er genervt, weil er gehen will», sagt Tobias. "Ja, ich weiss, er versteht nichts», antwortet die Assistenzärztin. Jemand fügt hinzu, dass der Dolmetscher schon da war. Dann entscheidet die junge Ärztin: «Die Unterassistenten müssen Rücksprache mit dem Oberarzt nehmen, ob der Dolmetscher kommen soll oder nicht.» Keine Reaktion, nur ein leichtes Nicken. Dann wechselt die Diskussion zu einem anderen Patienten. Wurde dieses «Setting» von den Studierenden gut gemeistert? Dem Expertinnenauge der Bildungsverantwortlichen Andrea Bärlocher entging es jedenfalls nicht.

\section{Jenseits von Rollen und Hierarchien}

13.30 Uhr: zurück im ZIPAS-Büro für die Reflexion. Andrea Bärlocher fragt: «Gibt es ein Setting, das eurer Meinung nach heute Morgen nicht optimal verlaufen ist?» Niemand reagiert. Die Expertin hilft: «Als ihr den möglichen Einsatz eines Dolmetschers erwähnt habt, habe ich eine klassische Rollenverteilung beobachtet. Wie schätzt ihr das Setting ein, und was habt ihr am Schluss für diesen Patienten entschieden?» Jeder erklärt seine Sicht auf den Umgang mit der Situation und seine Reaktion in dem Moment. Der Facilitator Pflege interveniert: "Ich habe darauf gewartet, dass jemand sagt: 'Der Patient braucht einen Dolmetscher', aber das kam nicht.» Frank Schäfer, der Oberarzt, dem die Studierenden stillschweigend die Entscheidung überlas- 
Erste interprofessionelle Ausbildungsstation der Schweiz

2017 haben sich sechs Institutionen, darunter das Careum, die Universität Zürich, das USZ und die Zürcher Hochschule für Angewandte Wissenschaften ZHAW, zusammengeschlossen, um ZIPAS als erste interprofessionelle klinische Ausbildungsstation der Schweiz zu gründen. Das Projekt ist inspiriert vom schwedischen Modell, das seit den 1990er Jahren existiert. 2018 wurden die ersten beiden Pilotprojekte von ZIPAS an der Neurologischen Klinik des USZ durchgeführt. Ein Jahr später wurden am USZ die ersten Schweizer Ausbildungsstationen in Betrieb genommen (Klinik und Poliklinik für Innere Medizin und Klinik für Traumatologie). Während dreier bis vier Wochen übernimmt eine Gruppe von sechs bis sieben Studierenden (Ergotherapie, Physiotherapie, Medizin, Ernährungsberatung, Soziale Arbeit, Fachpersonen Gesundheit, Pflegefachpersonen), die sich in der Endphase ihres Studiums befinden, die Tagesstruktur und die Patientenversorgung. Die Gruppe gestaltet gemeinsam die Ziele und Interventionen für die Behandlung und führt Visiten bei den Patientinnen und Patienten durch. Erfahrene Berufsbildnerinnen und -bildner beziehungsweise Kaderärztinnen und -ärzte aus den jeweiligen Berufsgruppen begleiten die Gruppe als «Facilitators» während des Einsatzes. Gegen Ende jeder Schicht und Woche reflektieren Studierende und Facilitators gemeinsam die interprofessionelle Zusammenarbeit. Weitere Informationen: www.zipas.ch

sen hatten, ergänzt: «Hier sieht man das Klischee der steilen Hierarchie: der Höchstrangige muss entscheiden. Ihr hättet untereinander entscheiden können, ob ein Dolmetscher nötig ist, euch überlegen, welche Berufsgruppen bei dieser Gelegenheit etwas zu übersetzen haben, und diesen selber organisieren können.» Aysel, eine angehende Pflegefachfrau, die nicht auf der Visite war, entgegnet: «Einen Dolmetscher organisieren gehört zu den Kompetenzen der Pflege, und ich hätte es gemacht, wäre ich zuständig gewesen. Aber ich kann verstehen, dass man in dieser Situation die Zustimmung 'von oben' einholen wollte, da diese Dienstleistung Kosten verursacht.» Für Aysel ist ZIPAS eine lehrreiche Erfahrung: sie sei über die jeweiligen Rollen hinausgewachsen und sehe die Ärztin oder den Arzt als

«Das Projekt sollte auch auf die Weiter- und Fortbildung abzielen, um so viele Gesundheitsprofis wie möglich zu erreichen.»

gleichberechtigtes Gegenüber, das nicht mehr, sondern anderes weiss. "Am Anfang dachte ich: 'Darf ich den Arzt so etwas fragen?' Bei ZIPAS habe ich gelernt, offen mit der Ärzteschaft zu sprechen. Wir, die Pflegenden, können den Ärztinnen und Ärzten auch etwas mitgeben. Wir haben Kompetenzen, die sie nicht haben.»

Bei der Reflexion geht es darum, das Setting zu beschreiben und zu analysieren und die verschiedenen Standpunkte zu sammeln, um eine Lösung zu finden, die beim nächsten Mal umgesetzt werden kann. «Die Studierenden müssen ihre eigenen Lösungen finden und die eigenen Ressourcen nutzen", betont Andrea Bärlocher. «Ich bin jedes Mal von den Aha-Erlebnissen beeindruckt, wenn sie erkennen, was sie anders hätten tun können. Sie haben ein grosses Verständnis füreinander und den Wunsch, die Zusammenarbeit zwischen
Die Patientin oder der Patient stehe immer im Mittelpunkt des Reflektierens: «Wie muss man kommunizieren und handeln, damit die Behandlung so effizient, angenehm und sicher wie möglich ist? Dazu ist es wichtig, zu verstehen, was der andere tut, und die richtigen Informationen im richtigen Moment weiterzugeben.»

\section{Vom Projekt zur festen Etablierung}

Bei der ZIPAS hat man Zeit, diese Settings zu dekonstruieren. Wie sieht es damit im realen Leben aus? «Unser Alltag ist zwar stressig, aber ZIPAS hilft uns, die Interprofessionalität im Hinterkopf zu behalten. In ruhigeren Momenten können wir darüber nachdenken", meint Aysel. Andrea Bärlocher räumt ein, dass die Umsetzung im Alltag nicht einfach ist: «Wenn die Studierenden in ihre Routine zurückkehren, die anders funktioniert als ZIPAS, besteht die Gefahr, dass das Erlebte verloren geht. Es braucht eine Struktur, um es im Alltag zu leben und nachhaltig zu verankern.»

Ein weiteres Hindernis ist, dass im Berufsalltag nicht alle Gesundheitsprofis eine interprofessionelle Ausbildung erhalten haben oder dafür sensibilisiert wurden. Das kann sich bei der Umsetzung des Gelernten als frustrierend erweisen. Aysel bestätigt: «Manche Kolleginnen und Kollegen in der Pflege bleiben unbewusst in alten Mustern stecken. Manchmal fehlt das Interesse.» Die Verantwortlichen und Facilitators von ZIPAS sind sich dessen bewusst. Sie bedauern, dass derzeit nur ein kleiner Prozentsatz der Studierenden von der Ausbildungsstation profitiert. "ZIPAS ist wie eine künstliche Werkstatt, die Zusammenarbeit zwischen den Berufen wird noch nicht von allen im Alltag gelebt und wahrgenommen», so Frank Schäfer. Die interprofessionelle Ausbildung müsse viel weiter verbreitet und zum festen Bestandteil in den Studiengängen aller Gesundheitsberufe werden. «Sie sollte auch für ältere Generationen geöffnet werden, die nicht mit dieser aktiv gelebten interprofessionellen Arbeit vertraut sind. Sie sollte nicht nur auf die Grundausbildung abzielen, sondern auch auf die Weiter- und Fortbildung, um so viele Gesundheitsprofis wie möglich zu erreichen", fügt er hinzu. Um ZIPAS längerfristig und umfangreich zu etablieren und zu einer festen Ausbildungsstation zu machen, braucht es jedoch die notwendigen finanziellen Mittel und Personalressourcen ebenso wie den politischen Willen. Andere Spitäler im Land haben bereits ihr Interesse bekundet.

\section{Literatur}

1 Institute of Medicine (US) Committee on Quality of Health Care in America. To Err is Human: Building a Safer Health System. Kohn LT, Corrigan JM, Donaldson MS, editors. Washington (DC): National Academies Press (US); 2000. PMID: 25077248. 